\title{
Automatic Measurement of Neutral Foot Posture Using Three-Dimensional Scanning
}

\author{
Chien-Hung Lin, Yan-Yu Lin \\ Department of Mechanical Engineering \\ Chinese Culture University \\ 55, Hwa-Kang Road, Yang-Ming-Shan, Taipei, Taiwan 11114, R.O.C.
}

\section{Article Info}

Article History:

Received July 22, 2021

Revised August 5, 2021

Accepted August 20, 2021

\section{Keywords: \\ 3D scanning \\ Neutral foot posture \\ Leg angle \\ Foot angle \\ Rearfoot angle}

Abstract

The Rearfoot Angle (RFA) is the most common method used for foot posture assessment, and it is also a method for evaluating the subtalar joint neutral foot posture. However, orthopedists and researchers often meet trouble for the measurements of the RFA and neutral foot posture due to lacking measurement with automation and objectivity. In general, the RFA was measured using a goniometer to align with manual markers on foot. The purpose of this study implements the automatic computing of leg angle, foot angle, and RFA by the foot model using Three-Dimensional (3D) scanning. This study aims to implement the automatic and objective computing of leg angle, foot angle, and RFA by the foot model using 3D scanning. The automatic calculation on leg angle, foot angle, and neutral foot posture has been created, tested, and validated completely in this study. Two algorithm methods are proposed to determine the midpoint on the leg or foot outline. The midline has been computed by linear regression through five midpoints. The leg and foot angles are calculated by the tibial and calcaneal midlines, respectively. Through the subject standing on eversion foot platforms, the neutral posture of the foot can be computed by the leg and foot angles determined by the 3D model scanned of the foot with various tilt angles. The determinations of the midpoint and midline have been demonstrated algorithm by MATLAB. Based on comparing with the goniometer measured, selecting the midpoint algorithm of the limit points and the lowest point methods to determine the tibial and calcaneal midlines would produce better results. The foot $3 \mathrm{D}$ scanning measurement proposed in this study has been tested and validated from the goniometer. This study can determine the leg angle, foot angle, and neutral foot postures for a subject with normal weight status but not suitable for obese weight status. This study can provide guidance for foot posture assessment and personal insoles design in the future.
\end{abstract}

\author{
Corresponding Author: \\ Chien-Hung Lin \\ 1jh13@ulive.pccu.edu.tw \\ Department of Mechanical Engineering \\ Chinese Culture University \\ 55, Hwa-Kang Road, Yang-Ming-Shan, Taipei, \\ Taiwan 11114, Republic of China
}

This work is an open-access article and licensed under a Creative Commons Attribution-ShareAlike 4.0 International License (CC BY-SA 4.0) .

\section{INTRODUCTION}

The human foot has 26 bones, 33 joints, 107 ligaments, 19 muscles, and tendons [1]. The feet touch the ground and support the weight of the body to overcome ground reaction forces whenever a person stands, walks, and runs. Poor foot posture can cause problems to all body parts [2], including the hips, spine, lower back, shoulders, and neck. There are some methods for foot posture assessment used, including the evaluation of the Rearfoot Angle (RFA) [3-7], medial longitudinal arch angle [5-
6], navicular drop [8], footprint (arch index and malleolar valgus index) [6],[9], and radiographic data [10-11]. The RFA is the most commonly used method for foot posture assessment, and it is also a method for evaluating the Subtalar Joint (STJ) neutral foot posture [12-13]. Several researchers have presented by using the goniometer [3-5], photogrammetry [6-7], and digital radiography [10-11] to measure RFA to obtain a neutral posture of the foot. Nevertheless, the measurement of RFA and neutral foot posture often meet difficulties for orthopaedists and 


\section{Indonesian Journal of Electronics, Electromedical Engineering, and Medical Informatics (IJEEEMI) IJEEMI, Vol. 3, No. 3, August 2021, pp. 84-92

researchers, because these measurement methods lack automation and objectivity.

For generally traditional RFA measurement, the goniometer is the most normally used [3-5]. The subject stands in a relaxed bilateral stance with their feet aligned straight ahead and shoulder-width apart. Through clinician palpating, four manual markers are used to conduct the bisection lines of the tibia and calcaneus; respectively; thus, the RFA is measured by the goniometer as the acute angle between the bisection lines of the tibia and calcaneus. The manual method for marking and measuring cannot provide an objective and automatic measurement to determine RFA. In terms of photogrammetric measuring RFA [6-7],[14], digital image processing is used instead of the goniometer to determine the two lines connected by three marks that are manually marked on the rearfoot in advance. The RFA is an angle between two lines. The method provides high reliability and is independent of RFA calculated. However, the examiner clinically determines drawing markers on the calcaneus and lower leg. As to digital radiography [1011], the resting calcaneal stance position is measured by both frontal plane radiographs, and the result is close to clinical goniometric measured. Nevertheless, the radiation exposure incurred during X-ray examination may pose health risks to patients. Although the radiation level is set to low, these rays are harmful, and their effects are not negligible. Lin et al. [13],[15] presented that RFA is determined by using image processing to compute the calcaneus and tibia's midlines automatically.

The study on the RFA measured by the goniometer has been carried out by Jonson and Gross [3], Genova and Gross[4], and Langley et al.[5]. On the whole, their measuring method was conducted by manually and subjectively marking four markers on the foot, including the distal calcaneal mark of the first mark at the base of the calcaneus, the proximal calcaneal mark of the second mark at $3 \mathrm{~cm}$ above the first mark, the distal leg mark of the third mark at $6 \mathrm{~cm}$ above the second mark, and the proximal leg mark of the fourth mark at $8 \mathrm{~cm}$ above the third mark. Then the calcaneal bisection line was the first and second marks connected, and the tibial bisection line was the third and fourth marks connected. Lin et al. [15] proposed to automatically determine the feature points on the profiles of the lower leg and Achilles tendon by using the edge detection of image processing, then the midpoint was calculated by the feature points, and the tibial and calcaneal midlines were performed by linear regression from five midpoints. Moreover, Lin et al. [13] also presented using image processing to automatically calculate the midline of the tibia and calcaneus at various tilt angles by capturing rearfoot images of a subject standing on eversionable foot platforms. The tilt angle of the foot platforms denoted the STJ neutral position when the slopes of the midlines of the calcaneus and tibia were closer. As to the Three-Dimensional (3D) scanner, the 3D scanning can achieve a 3D model that is almost the same as the entity [16]. In medicine, 3D scanning is widely used to rapidly create accurate geometric of the surface of the human body [17]. Banga et al. [18] used a commercial system for handheld 3D scanning, a portable handheld scanner (Artec EVA Scan), to conduct the leg model building, and used
Rapid Prototyping (RP) to complete the prototype model of the leg. Cha et al. [19] employed a 3D scanner of Artec Eva to obtain the patient's lower leg for designing the ankle-foot orthosis. It is sufficient to show that the foot model of the foot scanned is consistent with the profile of the foot entity.

This study aims to implement the automatic and objective calculation of RFA by the foot model using 3D scanning. There were two methods proposed to determine the midpoint on the outline of the leg or foot. The results of the two methods calculated were compared with those of the goniometer measured. In addition, the neutral posture of the feet was achieved by calculating RFA with various tilt angles at the foot platforms for the subject standing. Therefore, this study has a high level of practicality and usability for evaluating foot health and orthosis applications.

\section{MATERIALS AND METHODS}

To establish an objective measurement for a neutral posture of the foot, the foot of a subject was scanned with an engineering 3D scanner. By capturing information of the 3D foot model, the midpoint on the outline was automatically calculated and midlines of the tibia and calcaneus were determined. Through the subject standing on eversion foot platforms with various tilt angles, the RFA was calculated to achieve the assessment of the neutral posture of the foot. Fig. 1 is a flowchart of 3D scanning to determine the neutral posture of the foot.

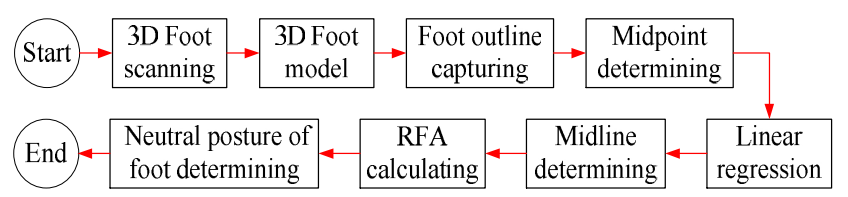

Fig. 1. Flowchart for 3D scanning to determine the neutral posture of the foot

\section{A. Subject}

In the experiment, there were three subjects: A subject was a 50 -year-old man with a height of $173 \mathrm{~cm}$, weight of $64 \mathrm{~kg}$, and BMI of 21.4, B subject was a 36-year-old woman with a height of $163 \mathrm{~cm}$, weight of $58 \mathrm{~kg}$, and BMI of 21.8 , and C subject was a 22-year-old man with a height of $174 \mathrm{~cm}$, weight of $95 \mathrm{~kg}$, and BMI of 31.4. All subjects were asymptomatic and free from injury and any known or visible skeletal abnormality that may have altered foot structure. Furthermore, the subjects also excluded histories of previous foot surgeries (such as trauma or fractures), neuropathies, obesity and musculoskeletal disorders (such as arthritis, tendinitis, bursitis, ankylosing spondylitis, and heel spurs). All subjects signed a statement of informed consent and the study was approved by the Ethical Review Approval National Taiwan University.

\section{B. 3D Scanning Foot}

In general, based on the classification of operation 3D scanners can be divided into two modes of fixed and handheld. Since the scanned object is a human's feet and lower legs, not an inanimate object, the handheld 3D scanner of Artec Eva Lite (Artec3D, Luxembourg, Luxembourg) was used in scanning. The Artec Eva Lite can capture a high accuracy of up to $0.1 \mathrm{~mm}$

\section{Accredited by Ministry of Research and Technology /National Research and Innovation Agency, Indonesia Decree No: 200/M/KPT/2020}




\section{Indonesian Journal of Electronics, Electromedical Engineering, and Medical Informatics (IJEEEMI) IJEEMI, Vol. 3, No. 3, August 2021, pp. 84-92

and a resolution of up to $0.5 \mathrm{~mm}$. The scanner can be operated at distances between $0.4 \mathrm{~m}$ and $1 \mathrm{~m}$ from the object, capturing up to 16 frames per second, each of which is automatically aligned in real-time. The set-up of the 3D scanning foot is shown in Fig. 2. The evaluation system comprises a $3 \mathrm{D}$ scanner, a computer, an eversion foot platform, and evaluation programs. For 3D scanning foot starting, a subject stands on the eversion foot platform with his feet shoulder-width apart to keep longitudinal axes of the feet parallel to each other. The Artec Studio software is launched when beginning the 3D scanning feet. The 3D scanner has a scan angle of 360 degrees around the leg and foot and lasts for $90 \mathrm{~s}$, and the scanner to achieve a complete 3D model of the lower leg. After erasing unneeded data, fusion, and mesh simplification, the $3 \mathrm{D}$ foot model is completed and saved as an STL file for subsequent use.

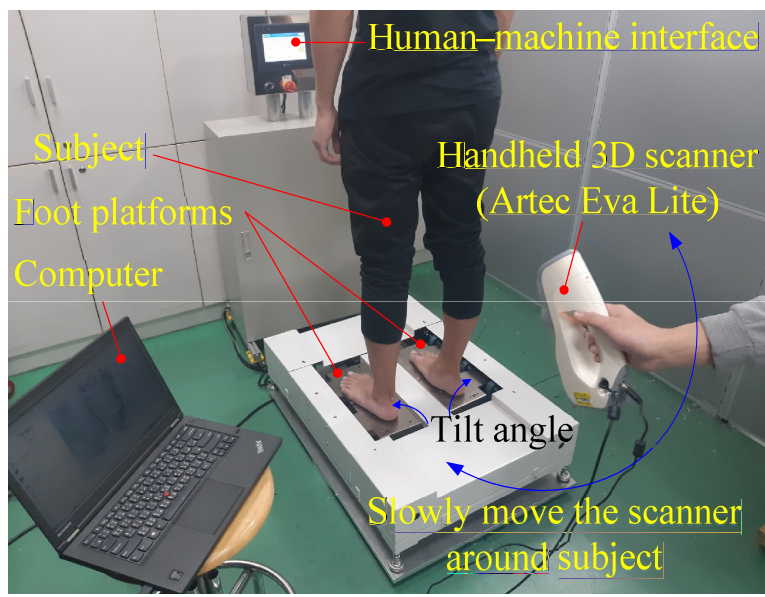

Fig. 2. Evaluation system for set-up of the 3D scanning foot

\section{Outline of the Lower Leg and Foot}

The STL file of a 3D foot model only describes the surface geometry of a 3D object. Through the G-code file converted from the slicing software, the coordinate data on the outline of the 3D foot model can be obtained. A G-code file is the most widely used for numerical control programming language, which is a document file with a list of coordinates and commands for the 3D printer to read. In our experiment, the STL file of the 3D foot model is loaded in Cura software, an opensource 3D printer slicing application, and scaled down to 0.25 units to calculate and display effectively. This application slices the foot model into hundreds of flat horizontal layers based on the settings. When the "spiralize the outer contour option" is checked on in the expert settings of Cura, the foot model does not generate any filling only draws the contour of the model. The outline of the foot at someone's height can be captured with the corresponding layer of the foot model by the G-code file. Then, the G-code file has imported into Microsoft Excel, which records the outline coordinates of each layer as a corresponding worksheet.

To evaluate the tibial and calcaneal midlines on the $3 \mathrm{D}$ foot model, the model has segmented into the lower leg and calcaneus portions, namely the upper and lower portions, respectively. Fig. 3 displays a schematic of the method used for distinguishing between the lower leg and calcaneus segments, which is modified from the method proposed by Genova and Gross [4]. The bottom point of the lower portion is at the base of the calcaneus and $2 \mathrm{~cm}$ above the footplate, and the length of the lower region is $3 \mathrm{~cm}$. The bottom point of the upper portion is 6 $\mathrm{cm}$ above the top of the lower portion, and the length of the upper portion is $8 \mathrm{~cm}$. In general, the two midpoints can determine a linear midline, but the accuracy of the midline depends on the correctness of the midpoint. To decrease the deviation of the calculating midline, there are five horizontal detecting layers on the upper and lower portions to divide the portion into four equal segments, which the midpoint has determined on the outline at detecting layers. As more detecting layers are taken, the dependence of the midline on the midpoint on the outline will decrease. However, too many detecting layers increase the processing time, so this study takes five detecting layers.

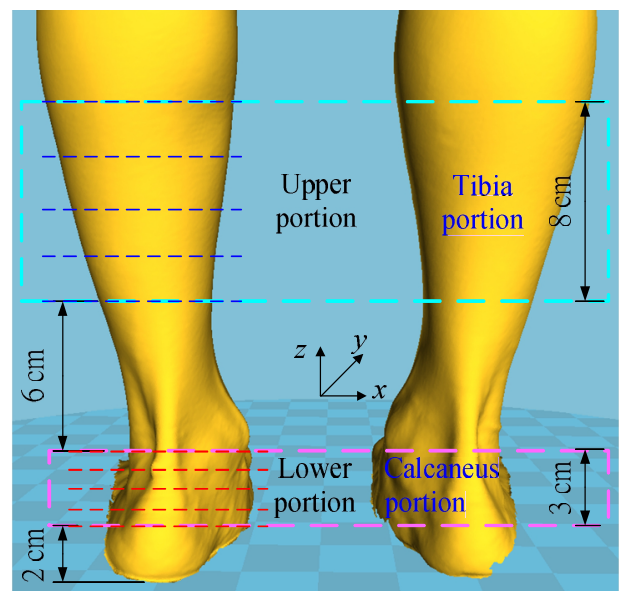

Fig. 3. Schematic of the method used for distinguishing between the lower leg (upper portion) and calcaneus portions (lower portion)

\section{Determine Midpoint on Outline of the Lower Leg and Foot}

In this study, the calculating midpoint on the outline has developed by MATLAB (MathWorks Inc., MA, USA). Based on the detecting layers on the lower leg and calcaneus portions, the coordinate data of the outline at the corresponding worksheet in Excel (Microsoft Office 2013, Microsoft Corp., Redmond, WA, USA) is imported into the program of MATLAB. There are two methods proposed for calculating the midpoint on the outline of the lower leg and foot as shown in Fig. 4. Method 1, the inflection points/ the limit points method, is to calculate the midpoint based on the left and right points on the outline. Depending upon calcaneus and lower leg portions, the midpoint calculated has different but to be similar to the clinical drawing points on the foot. For the calcaneus portion, the midpoint is calculated by the inflection points $L\left(x_{l}, y_{l}\right)$ and $R\left(x_{r}, y_{r}\right)$ on the outline at left and right sides near calcaneus, then the $\mathrm{x}$ coordinate of the midpoint is calculated $\left(x_{l}+x_{r}\right) / 2$. An inflection point is a point on the outline at which the curving changes and the second-order derivative is zero. The finite difference approximation of the second-order derivative of the outline at the point $P_{i}$ can be expressed as (1): 


\section{Indonesian Journal of Electronics, Electromedical Engineering, and Medical Informatics (IJEEEMI) IJEEMI, Vol. 3, No. 3, August 2021, pp. 84-92}

$$
\frac{y_{i+1}-2 y_{i}+y_{i-1}}{\left(x_{i+1}-x_{i}\right)\left(x_{i}-x_{i-1}\right)}, i=2,3,4, \ldots, n-1,
$$

where $\left(x_{i-1}, y_{i-1}\right),\left(x_{i}, y_{i}\right)$, and $\left(x_{i+1}, y_{i+1}\right)$ represent the $x$ and $y$ coordinates of the continue points $P_{i-1}, P_{i}$, and $P_{i+1}$ on the outline, respectively. The inflection points $\mathrm{L}$ and $\mathrm{R}$ are determined by the finite difference method from the outline at the left and right sides near calcaneus, respectively. For the lower leg portion, the midpoint has calculated by the limit points $L^{\prime}\left(x_{l}^{\prime}, y_{l}^{\prime}\right)$ and $R^{\prime}\left(x_{r}^{\prime}, y_{r}^{\prime}\right)$ on the outline at the leftmost and rightmost sides, then the $\mathrm{x}$ coordinate of the midpoint is equal to $\left(x_{l}{ }^{\prime}+x_{r}{ }^{\prime}\right) / 2$. The $x_{l}^{\prime}$ and $x_{r}{ }^{\prime}$ are the x coordinate at the leftmost and rightmost sides on the outline, respectively. Another method, Method 2, the lowest point method, is to calculate the midpoint based on the lowest point of the outline. The $x$ coordinate of the midpoint, $x_{\text {mid }}$, is equal to the corresponding point of minimum value of the $y$ coordinate on the outline.

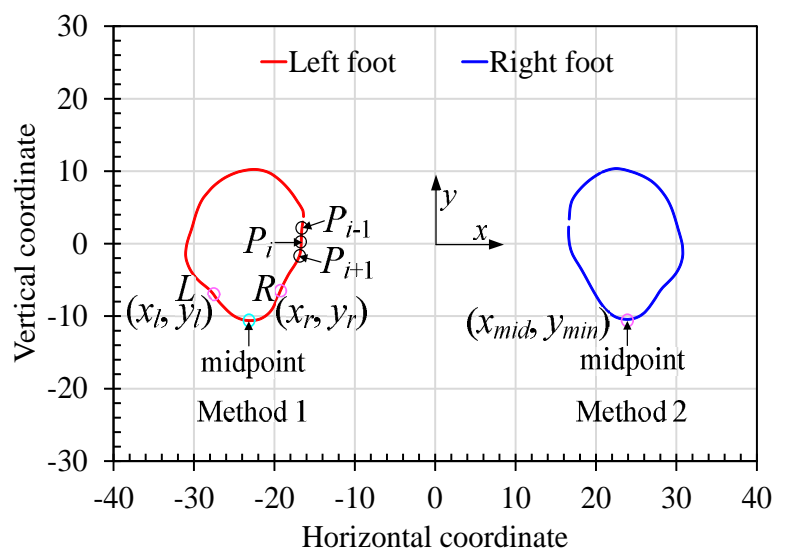

(a)

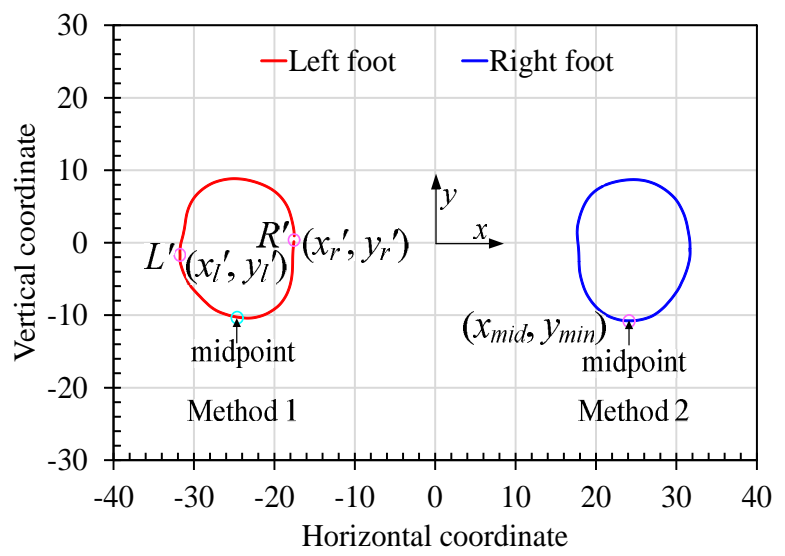

(b)

Fig. 4. Schematic of two methods for calculating the midpoint on the outline. (a) calcaneus portion and (b) lower leg portion

\section{E. Calculation of Foot and Leg Angles}

The study of calculating foot and leg angles, the midlines of the tibia and calcaneus are determined by calculating midpoints at five horizontal detecting layers on the upper and lower portions, respectively, which has demonstrated the development algorithm in MATLAB. Based on the $z$ coordinate of the corresponding height of each detecting layer and the $x$ coordinate of five midpoints calculate the midline through conducting linear regression for the least square method. However, the calculated midpoints on the outlines of the lower leg and foot inevitably yields some errors during data processing. The errors are from two sources: one is the accuracy and resolution of the scanner and the other is a scanned object. For the post-process of the scanning software, the scanning result is represented using free-form, unstructured threedimensional data, usually in the form of a point cloud or a triangle mesh. The accuracy of a 3D scanning instrument relates to the error by which the scan data points deviate from the actual location of the point with respect to the object, including the subject moving the foot during the scanning and the hair on the leg. Moreover, the outline of the completed 3D foot model converted by slicing software maybe produce some errors due to the conversion process. The optimum linear regression method has proposed in this study to enhance the relationship between data points and a linear line, which uses the adjacent points of the original data points to replace the previous data points for linear regression. To determine that the linear regression line with the coefficient closest to 1 is the midline. The adjacent points are theoretically continuous and very close. By adding the midpoints in the upward and downward layers on the five detection layers, the determination coefficient for calculating the midline by the linear regression method is improved. Fig. 5 shows the schematic diagram of adding the upward and downward layers in the original detecting layer to calculate the midpoint on its outline. Note that Mio is the midpoint at the $i$ th horizontal detecting layer, and $M_{i}^{+}$and $M_{i}^{-}$are the midpoints on the upward and downward layers respectively, where $i=1,2,3$, 4 , and 5 . According to the five detecting layers, using linear least squares regression for the five data points of $D_{i}$ determines the midline, where $i=1,2,3,4$, and 5. Each data point, Di, has three possible midpoints $-M_{i}^{+}, M_{i}{ }^{o}$, and $M_{i}^{-}$. Thus, there are $3^{5}=243$ possible regression lines for the five data points when the optimal linear regression method is used. The midline $z=m x+$ $b$ is determined using the possible regression lines when its corresponding coefficient of determination $R^{2}$ is the nearest to one. This line is referred to as the optimum midline. Here, $\mathrm{m}$ is the slope of the midline; $b$ is the $z$ intercept (i.e., the value of $z$ when $\mathrm{x}=0$ ); and $x$ and $z$ are the horizontal and vertical axes, respectively. $R^{2}$ ranges from zero to one and is an indicator of the relationship between the data points and the fitting line, which presents the probability of data lying on the regression line. The value of one indicates that the regression line represents all the data. The foot and leg angles for the left and right feet are calculated based on the midlines of the calcaneus and lower leg, respectively:

$$
\begin{aligned}
& \left\{\begin{array}{l}
\theta_{\text {foot }} \text { or } \theta_{\text {leg }}=\tan ^{-1} m ; \quad m \geq 0 \\
\theta_{\text {foot }} \text { or } \theta_{\text {leg }}=\tan ^{-1} m+\pi ; m<0
\end{array}\right. \text { for the left foot, } \\
& \left\{\begin{array}{l}
\theta_{\text {foot }} \text { or } \theta_{\text {leg }}=\pi-\tan ^{-1} m ; m \geq 0 \\
\theta_{\text {foot }} \text { or } \theta_{\text {leg }}=-\tan ^{-1} m ; \quad m<0
\end{array}\right. \text { for the right foot. }
\end{aligned}
$$

\section{Accredited by Ministry of Research and Technology /National Research and Innovation Agency, Indonesia Decree No: 200/M/KPT/2020}




\section{Indonesian Journal of Electronics, Electromedical Engineering, and Medical Informatics (IJEEEMI) \\ IJEEMI, Vol. 3, No. 3, August 2021, pp. 84-92 \\ DOI: 10.35882/ijeeemi.v3i3.1}

\section{F. Neutral Posture of the Foot}

In the experimental process for calculating the neutral foot posture, the left and right feet of the subject are placed on the left and right eversion foot platforms, respectively. The tilt angle has varied from 0 degrees to 20 degrees with a step of 5 degrees on the eversion foot platforms, and the 3D foot model is built by conducting 3D scanning at various tilt angles. By determination of the midpoint on the outline and the optimum midline, the foot and leg angles are calculated using the 3D foot model at various tilt angles. The neutral posture of the foot appears on the angle of the foot and leg being equal.

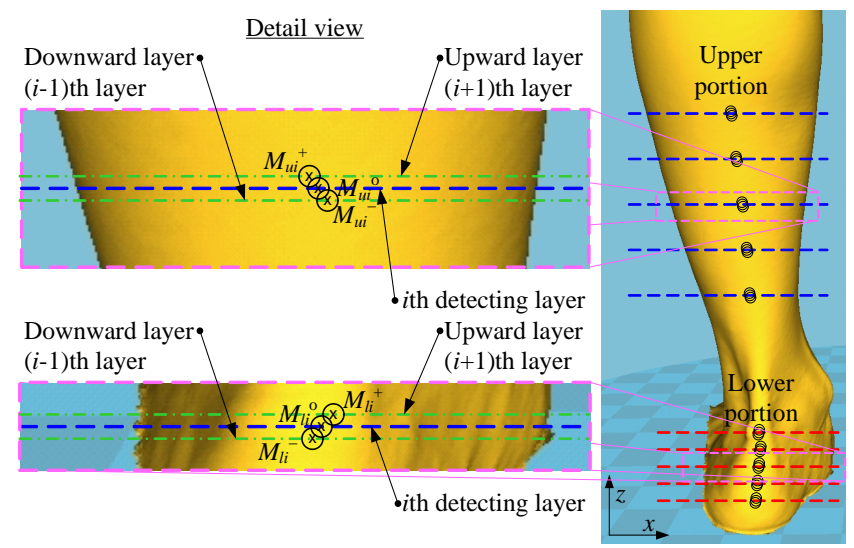

Fig. 5. Schematic diagram of adding the upward and downward layers in the original detecting layer to calculate the midpoint on its outline

\section{RESULTS}

\section{1) Foot Model by 3D Scanning}

For the Artec Eva Lite scanner, a subject stood on the eversion foot platforms, and then the scanner was slowly moved around the subject and kept proper distance between the subject and the scanner to achieve a complete 3D model of the lower leg. The complete 3D foot model from scanning took approximately 90 seconds that it significantly reduced scanning time to avoid a subject shaking or moving his feet while scanning. Fig. 6 displays the results of the $3 \mathrm{D}$ foot model obtained from the Artec Eva Lite scanner. The 3D foot model was exported as an STL file by scanning software so that it can be imported into the slicing software Cura later.

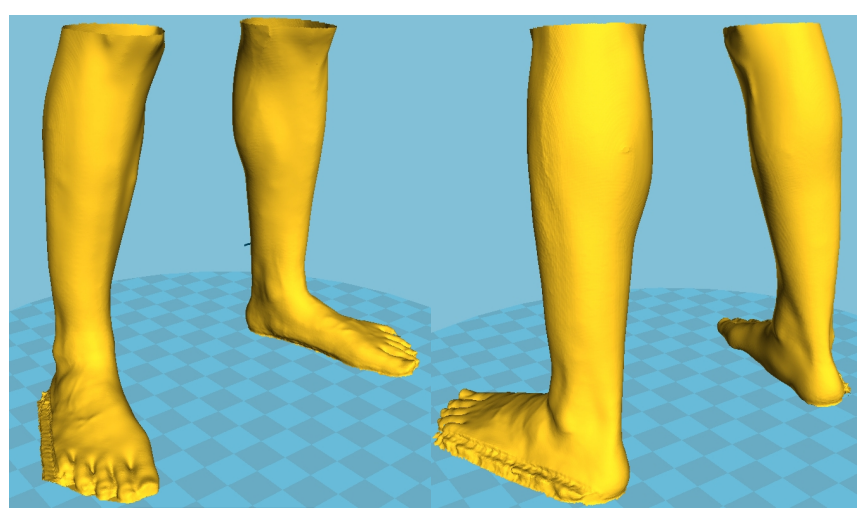

Fig. 6. 3D scanning foot model from Artec Eva Lite scanner

\section{2) Calculating Midlines of the Tibia and Calcaneus}

To verify the accuracy of two methods of determining the midpoint on the outline, a polynomial outline was established as $y=0.01(x+1)(x-2)(x+3)(x-4)=0.01\left(x^{4}-2 x^{3}-13 x^{2}+14 x+24\right)$, where $x$ ranges from -5 to 5 with $x$ step of 0.02 . The inflection point of this polynomial occurs at $x=-1.0545$ and 2.0545 and the minimum value of $y$ occurs at $x=-2.1926$ and 3.1926. To verify the program of the midpoint determination, the results of the program showed that the inflection points are at $x=-1.0545$ and 2.0545 for Method 1 and the minimum points are at $x=-2.2$ and 3.2 for Method 2 as shown in Fig. 7. Program verification was correct in determining the inflection point but was a slight error in determining the minimum point. The reason for the error was that the polynomial outline is established by the $x$ step of 0.02 , and its minimum value $y=-0.24998$ occurs at $x=-2.2$ and 3.2. Fig. 8 shows the result of MATLAB performing the midpoint calculation on the outlines of the calcaneus and the lower leg portions. There were some differences in the calculation of midpoint between the two methods. The following section will discuss and compare the results of the midlines obtained by the two methods.

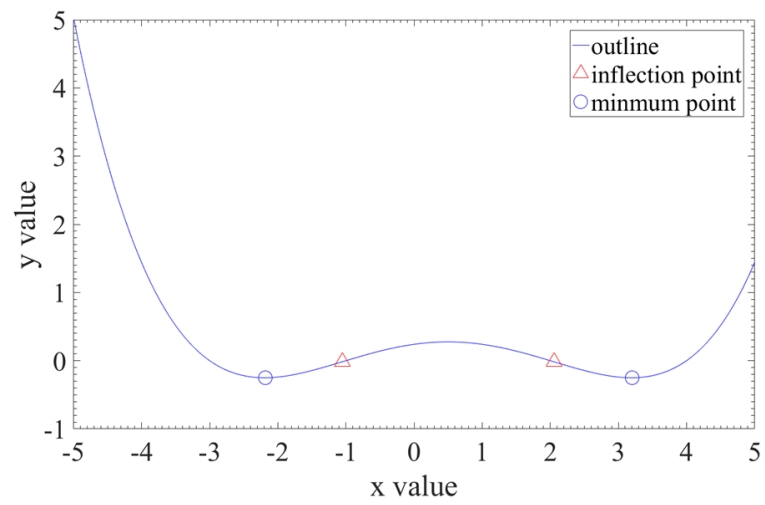

Fig. 7. Verification of the inflection and minimum points calculating with the MATLAB program

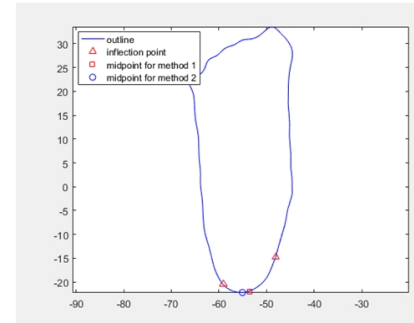

(a)

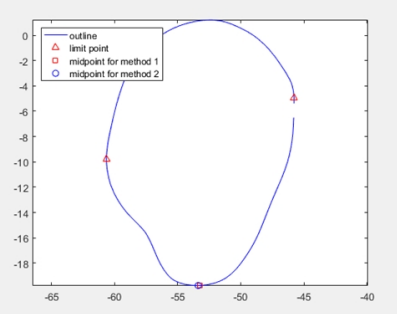

(b)
Fig. 8. The result of MATLAB performing the midpoint calculation. (a) the outline on the calcaneus portion (b) the outline on the lower leg portion

3) Determination Leg and Foot Angles

The midline was calculated by linear regression for the relationship between the $\mathrm{x}$ coordinate of five midpoints and the $z$ coordinate of the corresponding height of the detecting layers. The leg and foot angles were determined by the slopes of the tibial and calcaneal midlines, respectively. Fig. 9 presents the result of the calculation midline with Method 1 when the foot

\section{Accredited by Ministry of Research and Technology /National Research and Innovation Agency, Indonesia Decree No: 200/M/KPT/2020}

Journal homepage: http://ijeeemi.poltekkesdepkes-sby.ac.id/index.php/ijeeemi 


\section{Indonesian Journal of Electronics, Electromedical Engineering, and Medical Informatics (IJEEEMI) \\ IJEEMI, Vol. 3, No. 3, August 2021, pp. 84-92}

DOI: 10.35882/ijeeemi.v3i3.1

ISSN:2656-8624

platforms are at a tilt angle of 0 degrees. In the leg portion, the slopes of the tibial midline and the optimum midline were very close. The leg angles were 91.0 degrees and 95.4 degrees for the left and right tibial midlines, respectively. Furthermore, the leg angles were 91.0 degrees and 95.3 degrees for the left and right tibial optimum midlines, respectively. The leg angles calculated by the midline and optimum midline were almost the same. Furthermore, the determination coefficient of the tibial midline calculated by linear regression was only slightly increased for the optimum midline. In the foot portion, the slope of the calcaneal midline was a little different from that of the optimum midline. The foot angles were 79.4 and 80.9 degrees for the left and right tibial midlines, and 81.5 and 76.0 degrees for the left and right calcaneal optimum midlines, respectively. The foot angles calculated by the midline and optimum midline had some differences but little degree. However, the coefficient of determination of the midline of the calcaneus calculated by linear regression was increased significantly and approached 1 compared with its corresponding optimal midline. Fig. 10 demonstrates the result of the calculation midline with Method 2 at a tilt angle of 0 degrees on the foot platforms. Regardless of the leg or foot portion, the slopes of the tibial and calcaneal midlines were close to those of the optimum midlines. The leg and foot angles were 87.8, 82.1, 95.2, and 84.8 degrees for the left and right midlines, and 87.8, 83.9, 94.9, and 84.8 degrees for the left and right optimum midlines, respectively. All determination coefficients of the midlines calculated by linear regression were greater than 0.8 , and their corresponding optimum midlines approached 1.

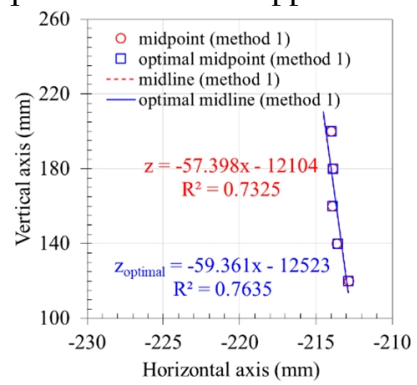

(a)

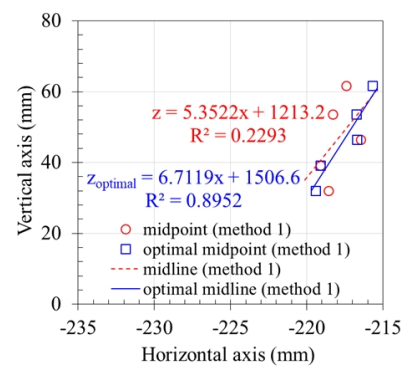

(c)

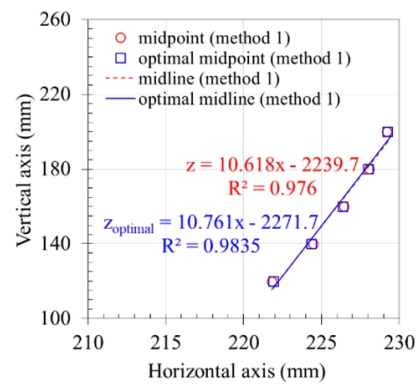

(b)

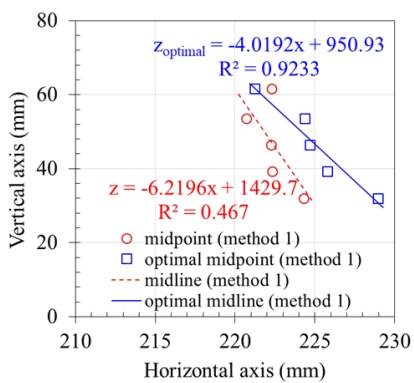

(d)
Fig. 9. The results of the midline calculated by Method 1 at a tilt angle of 0 degrees: (a) tibial midline of the left foot, (b) tibial midline of the right foot, (c) calcaneal midline of the left foot, (d) calcaneal midline of the right foot.

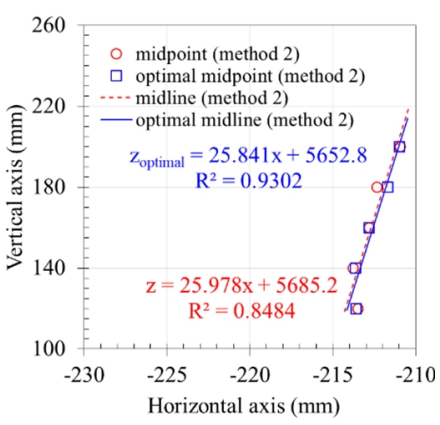

(a)

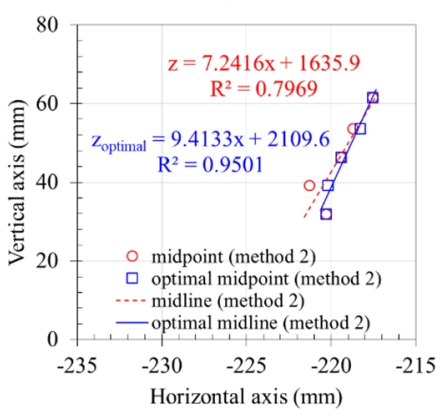

(c)

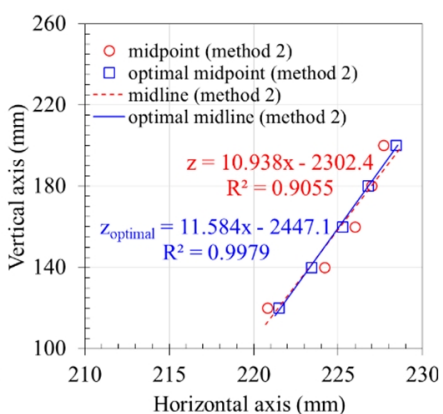

(b)

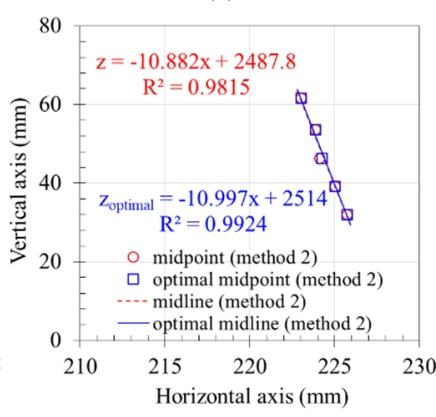

(d)
Fig. 10. The results of the midline calculated by Method 2 at a tilt angle of 0 degrees: (a) tibial midline of the left foot, (b) tibial midline of the right foot, (c) calcaneal midline of the left foot, (d) calcaneal midline of the right foot

Table I summarizes the results of the above data of the leg and foot angles. Compared with the two methods for calculating leg and foot angle, the angle difference of the leg or foot angle calculated by the two methods was about 3 degrees. Through the goniometer measuring the leg and foot angles were 90.1, 83.3, 92.4, and 86.4 degrees for left and right feet, respectively. Therefore, selecting Method 1 to determine the tibial midline in the upper portion and Method 2 to determine the calcaneal midline in the lower portion were able to be closer to the results of the goniometer measured. Moreover, RFA is equal to the difference between the leg and foot angles which provides the classification result of the foot posture. For this case in Table 1, based on the RFA results calculated from the 3D scanning, the foot posture of the subject was classified as the pronation, which is the same as the measurement result of the goniometer measured.

TABLE I. THE DETERMINATION OF LEG AND FOOT ANGLES BY METHOD 1 AND METHOD 2.

\begin{tabular}{|c|c|c|c|}
\hline \multicolumn{2}{|c|}{ Items } & Left (optimum) & Right (optimum) \\
\hline \multirow{2}{*}{ Method 1 } & Leg angle & $91.0^{\circ}\left(91.0^{\circ}\right)$ & $95.4^{\circ}\left(95.3^{\circ}\right)$ \\
\cline { 2 - 4 } & Foot angle & $79.4^{\circ}\left(81.5^{\circ}\right)$ & $80.9^{\circ}\left(76.0^{\circ}\right)$ \\
\hline & RFA & $11.6^{\circ}\left(9.5^{\circ}\right)$ & $14.5^{\circ}\left(19.3^{\circ}\right)$ \\
\hline \multirow{2}{*}{ Method 2 } & Leg angle & $87.8^{\circ}\left(87.8^{\circ}\right)$ & $95.2^{\circ}\left(94.9^{\circ}\right)$ \\
\cline { 2 - 4 } & Foot angle & $82.1^{\circ}\left(83.9^{\circ}\right)$ & $84.8^{\circ}\left(84.8^{\circ}\right)$ \\
\hline & RFA & $5.7^{\circ}\left(3.9^{\circ}\right)$ & $10.4^{\circ}\left(10.1^{\circ}\right)$ \\
\hline
\end{tabular}

4) Neutral Posture of the Foot

The subject stood on eversion foot platforms and was scanned by the Artec Eva Lite scanner to achieve a 3D foot model, in

\section{Accredited by Ministry of Research and Technology /National Research and Innovation Agency, Indonesia Decree No: 200/M/KPT/2020}




\section{Indonesian Journal of Electronics, Electromedical Engineering, and Medical Informatics (IJEEEMI) IJEEMI, Vol. 3, No. 3, August 2021, pp. 84-92

which the tilt angle of the foot platform was from 0 to 20 degrees with a step of 5 degrees. In the upper and lower portions the midpoints on the outlines of foot were calculated by Method 1 and Method 2, respectively. For subject A, Fig. 11 displays the comparison of $3 \mathrm{D}$ scanning and the goniometer results of the leg and foot angles for subject A.

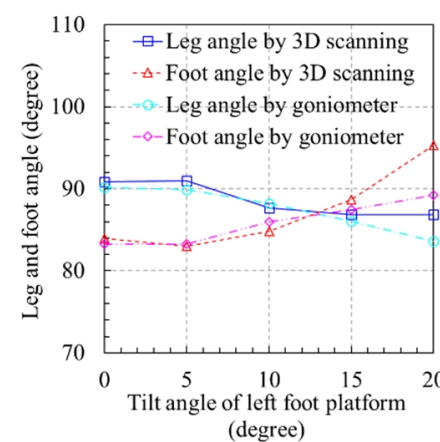

(a)

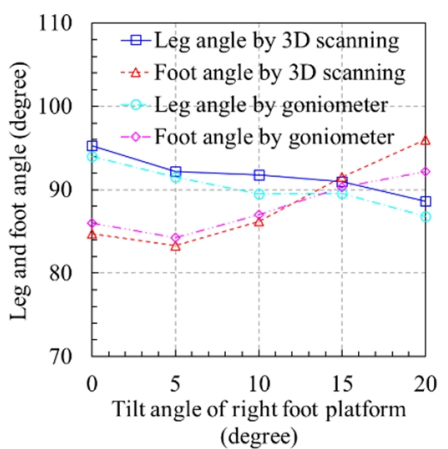

(b)
Fig. 11. Comparison of 3D scanning and the goniometer results of the leg and foot angles for subject A: (a) left foot and (b) right foot

According to the measuring result of the tilt angle on the foot platform at 0 degrees, the foot posture of subject $A$ was classified as the pronation. With the tilt angle of the foot platform increasing, the leg angle decreased but the foot angle increased, which the stance phase changed from pronation to supination. In addition, the results of the leg and foot angles obtained by using three-dimensional scanning and the goniometer were very close, but in tilt of foot platform of 20 degrees there was a greatly difference between the two method measured. The standing posture of the subject had been deformed as the larger tilt angle of the foot platform, which affected the result of the 3D scanning. For 3D scanning measured, a neutral foot posture that occurred the leg angle equal to the foot angle was estimated the tilt angles at about 13 and 15 degrees in the left and right feet, respectively. The result of the neutral foot posture obtained by the $3 \mathrm{D}$ scanning was the same as that of the goniometer measured.

Fig. 12 demonstrates the comparison of 3D scanning and the goniometer results of the leg and foot angles for subject B. Base on the measuring result of the tilt angle on the foot platform at 0 degrees, the foot posture of subject B was classified as the pronation. The trend of subject B measured was like that of subject A. A neutral foot posture was estimated as the tilt angles at about 16 and 15 degrees in the left and right feet, respectively. Fig. 13 presents the comparison of 3D scanning and the goniometer results of the leg and foot angles for subject $\mathrm{C}$. The foot posture of subject $\mathrm{C}$ was classified as the pronation duo to the measuring result of the tilt angle on the foot platform at 0 degrees. The results of the leg angle obtained by using threedimensional scanning and the goniometer had similar resultant values. Nevertheless, the foot angle obtained by using the threedimensional scanning was significantly different from measured by the goniometer.

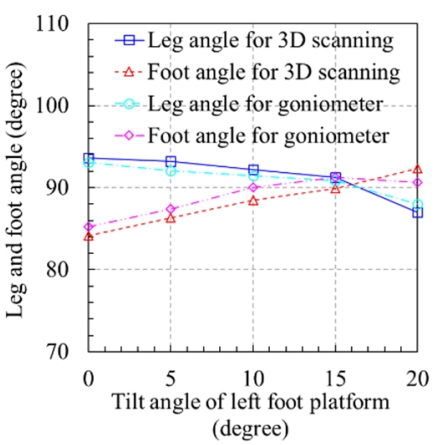

(a)

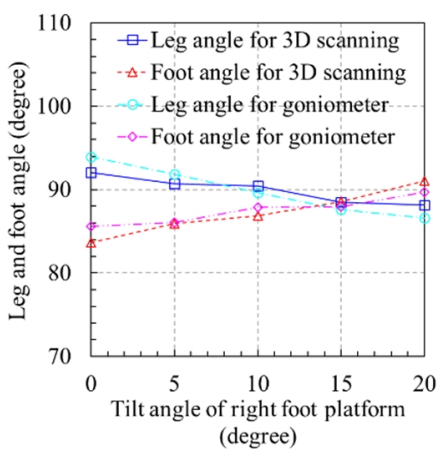

(b)
Fig. 12. Comparison of 3D scanning and the goniometer results of the leg and foot angles for subject B: (a) left foot and (b) right foot

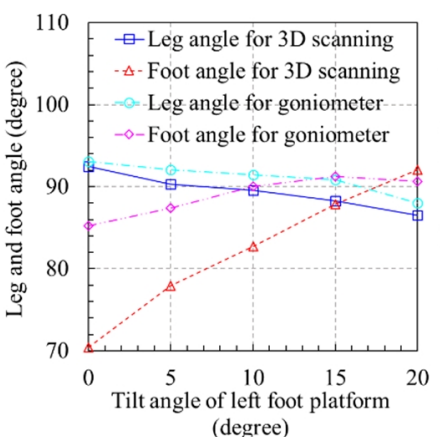

(a)

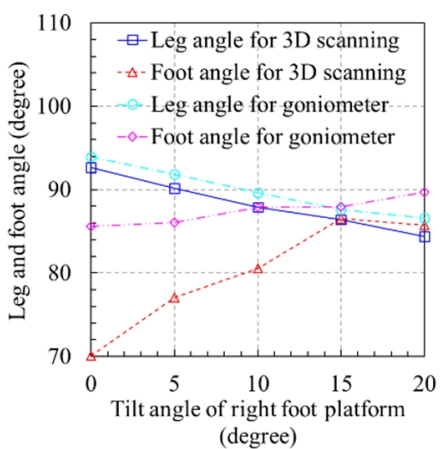

(b)
Fig. 13. Comparison of 3D scanning and the goniometer results of the leg and foot angles for subject C: (a) left foot and (b) right foot

The main reason was that the BMI of subject $\mathrm{C}$ is 34.1 and his weight status is obese, which caused fat near the calcaneus to affect the midpoint of the outline calculated by the threedimensional scanning foot model. Therefore, if a subject is too obese, the measurements in foot angle and neutral foot posture cannot be applied by the three-dimensional scanning method. On the other hand, the BMIs of subjects A and B were between 18.5 and 24 , a normal weight status, thus the 3D scanning method can be used to measure the leg angle, foot angle, and neutral foot posture.

\section{DISCUSSION}

It is rare to find studies that have conducted research on automated measurement for leg angles, foot angles, and RFA. Using manual marking and goniometer methods cannot provide the objectivity and accuracy required by medical data. The automatic calculation of neutral foot posture with 3D scanning technology has been created, test, and validated completely in this study. In addition to providing the calculation for neutral foot posture, this study also offers an automated determination of leg angle, foot angle, and RFA. In terms of the automated determination for the leg and foot angles, the calcaneal midline determined from this study was computed by capturing the foot profile data based on the actual position of the calcaneus. However, Lin et al. [15] used the edge detection of digital image processing to calculate the features of the Achilles tendon to 


\section{Indonesian Journal of Electronics, Electromedical Engineering, and Medical Informatics (IJEEEMI) IJEEMI, Vol. 3, No. 3, August 2021, pp. 84-92

determine the calcaneal midline, the midline calculated was higher than the actual position of the calcaneus. Furthermore, the result of edge detection is easily affected by the location of the camera, uniformity of the light source, and background. Therefore, the foot angle calculated in this study was actually more accurate than that by digital image processing. In addition, the leg and foot angles calculated were validated by using the goniometer. As to the classification results of the foot posture, Mukhtar et al. [14] conducted that determination of the points of tibial and calcaneus is carried out manually. This study was not only capable of computing the tibia and calcaneus points automatically but also providing the classification results of the foot posture through the calculation of RFA.

In research of the neutral foot posture, Chen et al. [12] conducted an in vitro study to evaluate the subtalar joint neutral position by inserting pressure-sensitive films into the anterior and posterior articulation of the subtalar joint. There were four types of foot position, including dorsiflexion, plantarflexion, inversion, and eversion. The subtalar joint neutral position was determined present when maximum contact area was achieved within the articulation. The most approximate subtalar joint neutral position was in the foot position of 10 of abduction, 20 of dorsiflexion, and 10 of eversion. However, their method was only suitable for cadaver feet and not for human subject research. They proposed that subtalar joint neutral position was affected by various types of foot position, which was consistent with the results of this study. Lin et al. [13] demonstrated that assessment of the subtalar joint neutral position was achieved by rear foot images with various tilt angles on the foot platform. Their results were not validated by the goniometer, and the midline calculated was higher than the actual position of the calcaneus as the same as the Ref [15]. This study carried out the neutral foot posture on three subjects. For subjects with normal weight status, the neutral posture by the $3 \mathrm{D}$ scanning method can be obtained the results as similar as the goniometer measured.

\section{Conclusion}

Leg angle, foot angle, RFA, and neutral foot postures are commonly used to evaluate foot for orthopedists and researchers, although the lack of carrying out the assessment with methods should be developed in automation and objectivity. This study has proposed two methods for determining the midpoint on the outline of the leg or foot. The determination of the midpoint has been demonstrated algorithm by MATLAB. In addition, the midline has been computed by linear regression through five midpoints, and the algorithm is also developed using MATLAB. Based on comparing with the goniometer measured, selecting the midpoint algorithm of Method 1 and Method 2 to determine the tibial and calcaneal midlines respectively would carry out better results. Then, leg angle, foot angle, and RFA can be calculated by the tibial and calcaneal midlines. Through the subject standing on eversion foot platforms, the neutral posture of the foot can be computed by the leg and foot angles determined by the 3D model scanned of the foot with various tilt angles. The 3D scanning method on foot has been tested and validated to demonstrate the leg angle, foot angle, and neutral foot posture for a subject of normal weight status, but be not suitable for that of obese weight status. In the future, this study can provide for foot posture assessment and personal insoles design.

\section{ACKNOWLEDGEMENT}

The authors are grateful for the support of the Ministry of Science and Technology of Taiwan, R.O.C., by grant number MOST 107-2221-E-034-007. We also thank for Wallace Academic Editing to edit this manuscript.

\section{REFERENCES}

[1] D. L. France, "Human and nonhuman bone identification: a color atlas," CRC Press, 2008.

[2] R. B. Brewer and A. J. Gregory, "Ghronic lower leg pain in athletes: a guide for the differential diagnosis, evaluation, and treatment," Sports Health, vol. 4, no. 2, pp. 121-127, 2012.

[3] S. R. Jonson and M. T. Gross, "Intraexaminer reliability, interexaminer reliability and mean values for nine lower extremity skeletal measures in healthy naval midshipmen," J. Orthop. Sports Phys. Ther., vol. 25, no. 4, pp. 253-263, 1997.

[4] J. M. Genova and M. T. Gross, "Effect of foot orthotics on calcaneal eversion during standing and treadmill walking for subjects with abnormal pronation," J. Orthop. Sports Phys. Ther., vol. 30, no. 11, pp. 664-675, 2000.

[5] B. Langley, M. Cramp, and S. C. Morrison, "Clinical measures of static foot posture do not agree," J. Foot Ankle Res., vol. 9, no. 1, pp. 45-1-6, 2016.

[6] A. P. Ribeiro, F. Trombini-Souza, V. Tessutti, F. R. Lima, I. C. N. Sacco, and S. M. A. João, "Rearfoot alignment and medial longitudinal arch configurations of runners with symptoms and histories of plantar fasciitis," Clin., vol. 66, no. 6, pp. 1027-1033, 2011.

[7] L. D. M. Mendonça, N. F. N. Bittencourt, G. M. Amaral, L. S. Diniz, T. R. Souza, and S. T. Fonseca, "A quick and reliable procedure for assessing foot alignment in athletes," J. Am. Podiatr. Med. Assoc., vol.103, no. 5, pp. 405-410, 2013.

[8] R. G. Nielsen, M. S. Rathleff, O. H. Simonsen, and H. Langberg, "Determination of normal values for navicular drop during walking: a new model correcting for foot length and gender,' J. Foot Ankle Res., vol. 2, pp. 1-12, 2009.

[9] H. B. Menz, M. R. Fotoohabadi, E. Wee, and M. J. Spink, "Visual categorisation of the arch index: a simplified measure of foot posture in older people,” J. Foot Ankle Res., vol. 5, no. 10, pp. 1-7, 2012.

[10] R. W. Mendicino, A. R. Catanzariti, S. John, B. Child, and B. M. Lamm, "Long leg calcaneal axial and hindfoot alignment radiographic views for frontal plane assessment," J. Am. Podiatr. Med. Assoc., vol. 98, no. 1, pp. 75-78, 2008.

[11] B. M. Lamm, R. W. Mendicino, A. R. Catanzariti, and H. J. Hillstrom, "Static rearfoot alignment," J. Am. Podiatr. Med. Assoc., vol. 95, no. 1, pp. 26-33, 2005.

[12] Y. X. Chen, G. R. Yu, J. Mei, J. Q. Zhou, and W. Wang, "Assessment of subtalar joint neutral position: a cadaveric study," Chin. Med. J., vol. 121, no. 8, pp. 735-739, 2008.

[13] C. H. Lin, C. C. Yeh, and Z. H. Qiu, "Assessment of subtalar joint neutral position: study of image processing for rear foot image," Proceedings of the World Congress on Engineering, July 5-7, London, vol. II, pp. 3-7, 2017.

[14] H. Mukhtar, D. Rahmawati, D. K. Silalahi, L. Novamizanti, M. R. Ghifari, A. A. A. Dzikri, F. F. Rachman, and A. A. Khatami "Fast algorithm to measure the types of foot postures with anthropometric tests using image processing", Indones. J. Electr. Eng. Comput. Sci., vol. 2, no. 1, pp. 48$59,2020$. 
[15] C. H. Lin, Z. H. Qiu, and C. C. Yeh, "Image processing for rear foot image evaluating leg and foot angles," Measurement, vol. 126, pp. 168-183, 2018.

[16] Y. Dessery, J. Pallari "Correction: Measurements agreement between low-cost and high-level handheld 3D scanners to scan the knee for designing a 3D printed knee brace," PLoS One, vol. 13, no. 4, pp. e0196183-1-14, 2018.

[17] L. Hale, E. Linley, and D. M. Kalaskar, "A digital workflow for design and fabrication of bespoke orthoses using 3D scanning and 3D printing a patient-based case study," Sci. Rep., vol. 10, pp. 7028, 2020.

[18] H. K. Banga, P. Kalra, R. M. Belokar, and R. Kumar, "Scanning and rapid prototyping of foot drop patient's leg," 14th International Conference on Humanizing Work and Work Environment, 2016.

[19] Y. H. Cha, K. H. Lee, H. J. Ryu, I. W. Joo, A. Seo, D. H. Kim, and S. J. Kim, "Ankle-foot orthosis made by 3D printing technique and automated design software," Appl. Bionics. Biomech., vol. , no. , pp., 2017.

Accredited by Ministry of Research and Technology /National Research and Innovation Agency, Indonesia Decree No: 200/M/KPT/2020 